\title{
Hereditary Fallopian Tube Carcinoma
}

National Cancer Institute

\section{Source}

National Cancer Institute. Hereditary Fallopian Tube Carcinoma. NCI Thesaurus. Code C40455.

Fallopian tube carcinoma that has developed in relatives of patients that have a history of fallopian tube carcinoma. 